OPEN ACCESS

Edited by:

Aixin Yan,

The University of Hong Kong,

Hong Kong

Reviewed by:

Adelaide Almeida,

University of Aveiro, Portugal

Yongping $X u$,

Dalian University of Technology, China

${ }^{*}$ Correspondence:

Shuai Le

leshuai2004@qq.com

Fuquan $\mathrm{Hu}$

hufuquan2009@yahoo.cn

Specialty section:

This article was submitted to Antimicrobials, Resistance

and Chemotherapy,

a section of the journal

Frontiers in Microbiology

Received: 27 November 2019

Accepted: 14 February 2020

Published: 04 March 2020

Citation:

Yang Y, Shen W, Zhong $Q$, Chen Q, He X, Baker JL, Xiong K,

Jin X, Wang J, Hu F and Le S (2020) Development of a Bacteriophage Cocktail to Constrain the Emergence

of Phage-Resistant Pseudomonas aeruginosa. Front. Microbiol. 11:327.

doi: 10.3389/fmicb.2020.00327

\section{Development of a Bacteriophage Cocktail to Constrain the Emergence of Phage-Resistant Pseudomonas aeruginosa}

\author{
Yuhui Yang', Wei Shen², Qiu Zhong ${ }^{3}$, Qian Chen', Xuesong He', Jonathon L. Baker ${ }^{6}$, \\ Kun Xiong ${ }^{1}$, Xiaoling Jin', Jing Wang ${ }^{1}$, Fuquan Hu${ }^{1 *}$ and Shuai Le ${ }^{1 *}$
}

\author{
${ }^{1}$ Department of Microbiology, Army Medical University, Chongqing, China, ${ }^{2}$ Department of Medical Laboratory, The General \\ Hospital of Western Theater Command, Chengdu, China, ${ }^{3}$ Department of Clinical Laboratory, Daping Hospital, Army \\ Medical University, Chongqing, China, ${ }^{4}$ Biomedical Analysis Center, Army Medical University, Chongqing, China, ${ }^{5}$ The \\ Forsyth Institute, Cambridge, MA, United States, ${ }^{6}$ Genomic Medicine Group, J. Craig Venter Institute, La Jolla, CA, \\ United States
}

With the emergence of multidrug-resistant and extensively drug-resistant bacterial pathogens, phage therapy and other alternative or additional therapeutic modalities are receiving resurgent attention. One of the major obstacles in developing effective phage therapies is the evolution of phage resistance in the bacterial host. When Pseudomonas aeruginosa was infected with a phage that uses $\mathrm{O}$-antigen as receptor, phage resistances typically achieved through changing or loss of $\mathrm{O}$-antigen structure. In this study, we showed that dsRNA phage phiYY uses core lipopolysaccharide as receptor and therefore efficiently kills the O-antigen deletion mutants. Furthermore, by phage training, we obtained PaoP5-m1, a derivative of dsDNA phage PaoP5, which is able to infect mutants with truncated O-antigen. We then generated a cocktail by mixing phiYY and PaoP5-m1 with additional three wide host range P. aeruginosa phages. The phage cocktail was effective against a diverse selection of clinical isolates of $P$. aeruginosa, and in the short-term constrained the appearance of the phage-resistant mutants that had beleaguered the effectiveness of single phage. Resistance to the 5phage cocktail emerges after several days, and requires mutations in both wzy and migA Thus, this study provides an alternative strategy for designing phage cocktail and phage therapy.

Keywords: dsRNA bacteriophage, phage cocktail, Pseudomonas aeruginosa, phage resistance, antibiotic resis

\section{INTRODUCTION}

Pseudomonas aeruginosa is a common opportunistic pathogen that causes infections of the bloodstream, urinary tract, burn wounds, and is one of the major pathogens infecting the airways of cystic fibrosis patients and P. aeruginosa infections can be life-threatening (Jonckheere et al., 2018; Waters and Grimwood, 2018). Moreover, P. aeruginosa strains are frequently resistant to multiple classes of antibiotics (Lopez-Causape et al., 2018), and P. aeruginosa is a member of the ESKAPE pathogens (Boucher et al., 2009), which include six pathogens with well-recognized abilities to 
develop antibiotic resistance and cause deadly clinical outbreaks. With the emergence of multidrug-resistant isolates of $P$. aeruginosa (Sun et al., 2013; Lopez-Causape et al., 2018), phage therapy has received renewed attention (Forde and Hill, 2018; Jault et al., 2018; Kortright et al., 2019), and is a promising alternative approach for treating recalcitrant $P$. aeruginosa infections (Roach et al., 2017; Waters et al., 2017; Forti et al., 2018; Jault et al., 2018).

An optimal phage therapeutic agent necessitates some features: low immunogenicity, strictly lytic lifestyle, no toxins or antibiotic resistant genes, a wide host range against multiple isolates of the target pathogen, and the ability to constrain the emergence of phage-resistant mutants (Pires et al., 2017; Rohde et al., 2018; Kortright et al., 2019). The host range of a phage treatment can be expanded by incorporating several phages with different host-range specificities within a cocktail. The host range and killing efficiency of phage cocktails targeting $P$. aeruginosa have been studied in vitro and in vivo (Roach et al., 2017; Forti et al., 2018). Phage resistance is a somewhat more difficult problem to address, as bacteria have evolved a number of different mechanisms to defend themselves against bacteriophages, including prevention of phage adsorption and DNA injection, restriction enzymes, and CRISPR/Cas systems (Hyman and Abedon, 2010). These mechanisms are highly effective and emerge rapidly. For example, a 6-phage cocktail formulated against a broad host range of $P$. aeruginosa initially killed with great efficiency. As expected, phage-resistant mutants grew to a high density in vitro after only overnight incubation (Forti et al., 2018). However, in the mice and larvae infection model, the phage cocktail resistant mutants are not observed (Forti et al., 2018). When using animal models to evaluate the efficacy of phage therapy, phage resistance is rarely observed, because the resistant mutants usually have a fitness tradeoff, or the minor mutants might be cleared by the immune system. On the contrary, phage resistant is quite common in vitro (Kortright et al., 2019). However, in clinics, a growing number of patients infected with multidrug resistant bacteria are immunocompromized, such as AIDS patients, transplant recipients with a suppressed immune system, diabetic patients, et al. These patients might not be able to clear the minor phage resistant bacteria and result in the treatment failure. Thus, the emergence of phage resistance has been reaffirmed by experts in the field as a key issue regarding the feasibility of phage therapy (Rohde et al., 2018; Kortright et al., 2019).

Pseudomonas aeruginosa develops phage resistance through several mechanisms, including modification or loss of the O-antigen component of lipopolysaccharide (LPS), or glycosylation of its type IV pilus (Harvey et al., 2018; Shen et al., 2018). Previously, we identified two types of phageresistant $P$. aeruginosa mutants following infection with the dsDNA phages PaP1 or PaoP5, based upon colony pigmentation (Figure 1A). The mutant designated PAO1r-1, with a brown colony phenotype, contained a large chromosomal deletion including the genes galU and $h m g A$, which respectively, resulted in the complete loss of $\mathrm{O}$-antigen and the accumulation of characteristic brown-colored homogentisic acids, while conferring phage resistance (Le et al., 2014; Shen et al., 2018).
Meanwhile, the mutant designated PA1RG, with a white colony phenotype, contained mutations in $w z y$, an LPS biosynthesis gene, which resulted in a truncated $\mathrm{O}$-antigen structure and also conferred phage resistance (Li et al., 2018). Thus, it seems mutations resulting in changes to LPS structure are likely to be a major source of phage resistance in $P$. aeruginosa. These phage-resistant mutants arose at a combined high frequency of $\sim 10^{-5}$ in vitro (Shen et al., 2018) and should be considered when designing a phage cocktail for eventual use in the clinical setting.

In this study, a phage cocktail designed to constrain the emergence of these phage-resistant phenotypes in P. aeruginosa was developed. The phage cocktail was successful in that it had a broader host range against a panel of $P$. aeruginosa clinical isolates compared to any of the tested single phages, and constrained the emergence of the previously described phage resistance phenotypes.

\section{RESULTS}

\section{The dsRNA Phage phiYY Infects Phage-Resistant PAO1r-1 With Exposed Core LPS}

Previously, in $P$. aeruginosa PAO1, we identified two types of mutants that resist infection by the dsDNA phage, PaoP5 (Figure 1A). One of these, PAO1r-1 has lost the O-antigen and can be easily identified via a brown colony phenotype caused by the accumulation of homogentisic acids (Shen et al., 2018). To attempt to identify a phage that bypasses this resistance mechanism, and was able to kill PAO1r-1, a panel of lytic phages was examined (Table 1). While PAO1r-1 was resistant to dsDNA phages, including PaP1 (Lu et al., 2013), PaoP5 (Shen et al., 2016), and PaP8 (Table 1), the dsRNA phage, phiYY, was able to lyse PAOr-1 and form clear plaques (Figure 1B).

As stated above, PAO1r-1 carries a deletion in galU and therefore lacks the $\mathrm{O}$-antigen component of LPS, leaving the core oligosaccharides of LPS exposed on the cell surface (Choudhury et al., 2005; Le et al., 2014). Accordingly, phiYY is likely to utilize the LPS core oligosaccharide instead of $\mathrm{O}$-antigen as the receptor to initiate infection (Figure 2). Because PAO1r-1 contains a large chromosomal deletion, a mutant containing a single-gene disruption of galU (PAO1 $\Delta$ galU), as well as complement strains restoring galU in both genetic backgrounds (PAO1r-1::galU and PAO1 $\Delta$ galU::galU), were examined for susceptibility to phiYY and PaoP5. As shown in Figure 1C, phiYY lysed PAO1r1 and PAO1 $\Delta$ galU, but did not kill the galU complemented strains, indicating that loss of galU allows for phiYY infection. On the contrary, PaoP5 infected PAO1, PAO1r-1::galU and PAO1 $\Delta$ galU::galU, but not the PAO1 $\Delta$ galU mutant (Figure 1D), indicating that loss of galU prevents infection by PaoP5.

A phage adsorption assay was used to further investigate the binding of these phages to the bacterial strains. phiYY adsorbs to PAO1r-1 and PAO1 $\Delta$ galU with high efficiency, but cannot bind to PAO1 or the galU complement strains (Figure 1E). Meanwhile, phage PaoP5 efficiently adsorbs to PAO1 and the galU complement strains, but not PAO1r-1 or 
A
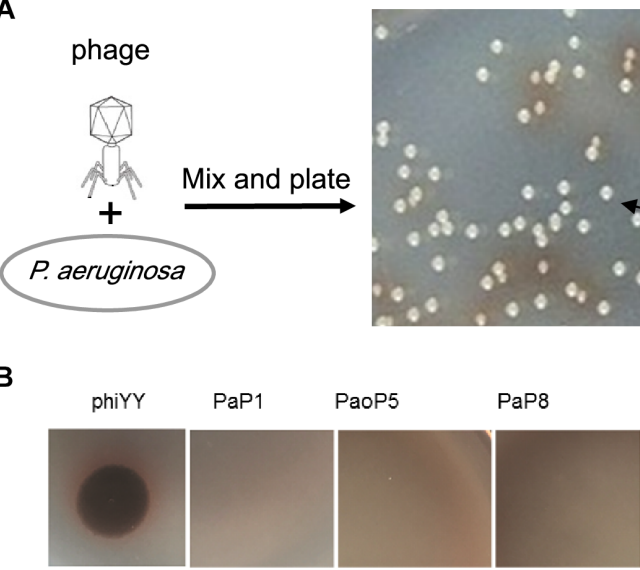

PAO1r-1

B

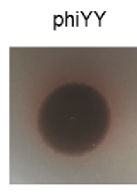

$\mathrm{PaP} 1$

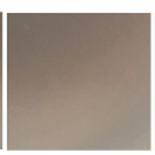

C

PAO1 PAO1r-1 PAO1r-1::galU PAO1 $\Delta$ galU PAO1 $\triangle$ galU:::galU

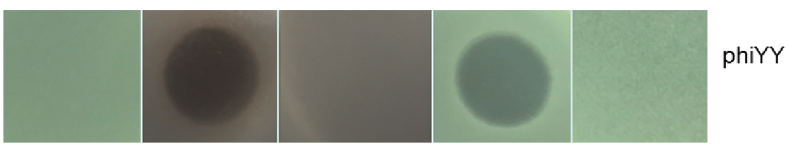

D

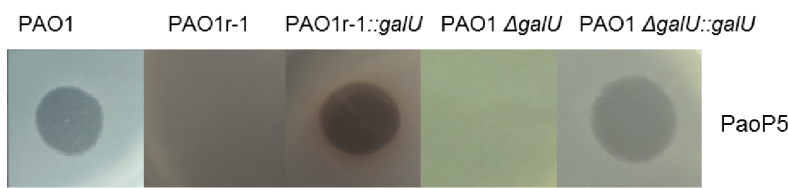

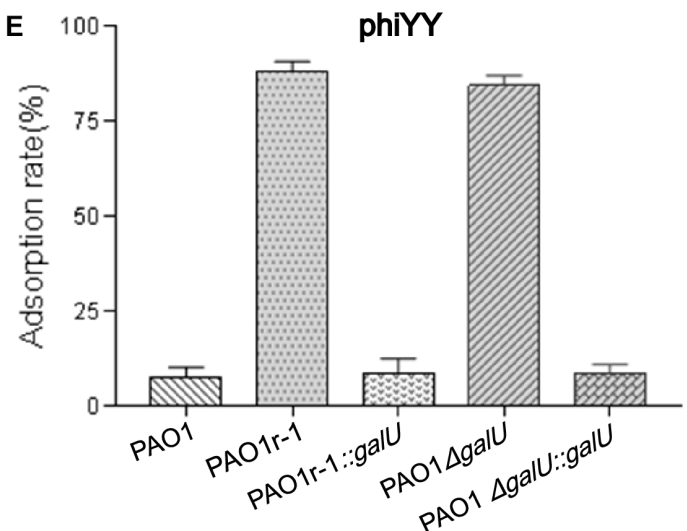

F $\left.{ }^{100}\right] \quad$ PaoP5

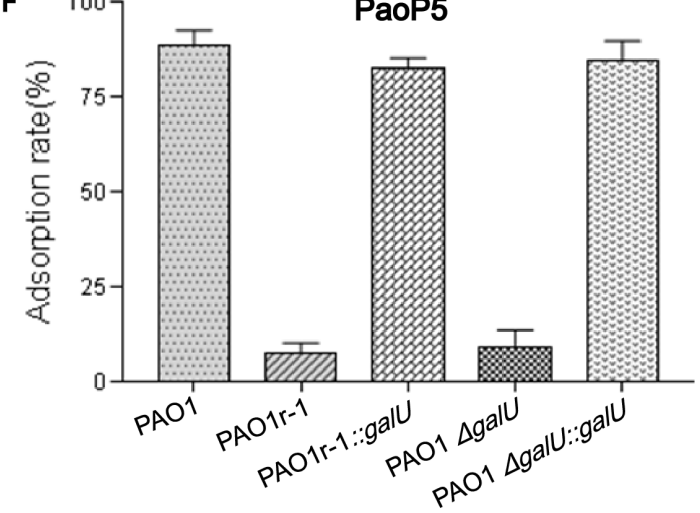

FIGURE 1 | dsRNA phage phiYY infects PAO1r-1 via exposed LPS core oligosaccharides (A): Colony phenotype of $P$. aeruginosa phage-resistant mutants, PAO1r-1 (brown) and PAO1w-1 (white). (B): Phage spot assay indicating that dsRNA phage phiYY, but not dsDNA phages PaP1, PaoP5, and PaP8, infects PAO1r-1. Phage spot assays (C,D) and phage adsorption assays (E,F) illustrating that phiYY infects and adsorbs to PAO1-derived strains without O-antigen, including PAO1r-1 and $\mathrm{PAO} 1 \triangle$ galU, while dsDNA phage PaoP5 infects and adsorbs to PAO1-derived strains with full O-antigen, such as PAO1 and the galU complement strains.

$(P<0.05$, one-way ANOVA, $n=3)$.

TABLE 1 | Bacterial strains and phages used in this study.

\begin{tabular}{|c|c|c|}
\hline Strain or phage & Description & Sources \\
\hline PAO1 & Wild type $P$. aeruginosa strain & Jacobs et al., 2003 \\
\hline PA1 & Wild type $P$. aeruginosa strain & Lu et al., 2015 \\
\hline PAO1r-1 & Phage-resistant mutant with brown pigment & Shen et al., 2018 \\
\hline PAO1r-1::galU & PAO1r-1 complemented with galU & This study \\
\hline $\mathrm{PAO} 1 \Delta$ galU & $\mathrm{PAO} 1 \Delta \mathrm{gal} U$ from the transposon library & Jacobs et al., 2003 \\
\hline PAO1 $\Delta$ galU::galU & $\mathrm{PAO1}$ 1 gal $\bigcup$ complemented with gal $U$ & This study \\
\hline PAO1w-1 & Phage-resistant mutant with a C1075T mutation in wzy & This study \\
\hline PAO1w-1::wzy & PAO1w-1 complemented with wzy & This study \\
\hline $\mathrm{PAO} 1 \Delta w z y$ & Knock out $\Delta w z y$ in PAO1 background & This study \\
\hline $\mathrm{PAO1} \Delta w z y:: w z y$ & PAO1 1 wzy complemented with wzy & This study \\
\hline $\mathrm{PAO} 1 \Delta m i g A$ & PAO1 $\Delta$ migA from the transposon library & Jacobs et al., 2003 \\
\hline $\mathrm{PAO} 1 \Delta \operatorname{mig} A \Delta w z y$ & Knock out wzy in PAO1 $\Delta$ migA & This study \\
\hline phiYY & dsRNA bacteriophage isolated from Southwest hospital sewage & Yang et al., 2016 \\
\hline PaP1 & dsDNA bacteriophage isolated from Southwest hospital sewage & Le et al., 2013 \\
\hline PaP8 & dsDNA bacteriophage isolated from Southwest hospital sewage & This study \\
\hline PaoP5 & dsDNA bacteriophage isolated from Southwest hospital sewage & Shen et al., 2016 \\
\hline PaoP5-m1 & PaoP5 mutant phage with a A715C mutation in orf75 & This study \\
\hline
\end{tabular}


PAO1 $\Delta$ galU (Figure 1F). Together, these data strongly indicate that the receptor for phiYY is the core oligosaccharide, while the receptor for PaoP5 is O-antigen. Therefore, phiYY may infect $P$. aeruginosa mutants lacking an $\mathrm{O}$-antigen that arise following infection by $\mathrm{O}$-antigen-binding phages, such as PaoP5.

\section{Training the dsDNA Phage PaoP5 to Infect White Mutants With Truncated LPS}

We previously identified PA1RG, a mutant of $P$. aeruginosa $\mathrm{PA} 1$, resistant to the dsDNA phage, $\mathrm{PaP} 1$, with a white colony phenotype (Li et al., 2018). This strain carries a C595T mutation in $w z y$, the $\mathrm{B}$-band $\mathrm{O}$-antigen polymerase, which results in a truncated $\mathrm{O}$-antigen with only one copy of the normallyrepeating sugar unit (Figure 2 ).

In this study, we isolated 5-phage-resistant PAO1 mutants with a white colony phenotype. Three out of these five isolates had mutations in $w z y$ as detected by Sanger sequencing of the PCR fragment amplified from the isolates. Two mutants had $a$ wzy C736G mutation and the third, designated PAO1w1, contained a wzy $\mathrm{C} 1075 \mathrm{~T}$ mutation, which resulted in a premature stop codon.

PAO1w-1 was highly resistant to the phages PaP1, PaoP5, PaP8, and phiYY (Figure 3A). To attempt to isolate a mutant phage that is able to effectively infect PAO1w-1, $10^{7}$ plaque forming units (pfu) of PaoP5 was mixed with PAO1w-1, and dozens of mutant phages that overcame resistance and lysed PAO1w-1 were observed. Five mutant phages were selected, purified and sequenced (Table 2). All 5-phage isolates that broke resistance of PAO1w-1 contained a A715C mutation in orf75, which was likely to be responsible for the expanded host range (Figure 3B). orf75 is located between the tail fiber gene and the baseplate gene. Though it is not annotated by blastp, Orf75 was identified as a structural protein by SDS-PAGE and highperformance liquid chromatography - mass spectrometry (Shen et al., 2016). Thus, the product of orf75 is highly likely to be a receptor-binding protein that is associated with host range.

One of the 5 mutant phages, designated PaoP5-m1, was selected for further study. Interestingly, unlike its parent strain, PaoP5, which did not infect PAO1w-1 or a single-gene inactivation of $w z y$ (PAO1 $\Delta w z y)$, PaoP5-m1 was able to adsorb and infect all of the PAO1-derived strains, regardless of the presence of full-length or truncated O-antigen (Figures 3C,D). To test the host range of PaoP5-m1 against a larger panel of PaoP5-resistant mutants, we selected 30 white colonies surviving PaoP5 infection across 3 biological replicates. Strikingly, PaoP5$\mathrm{m} 1$ infected all white mutants collected (Figure 3E), indicating that PaoP5-m1 is an excellent phage to eliminate $P$. aeruginosa mutants with truncated $\mathrm{O}$-antigen structures.

Interestingly, similar phage training was unable to generate a mutant phage that infected PAO1r-1, bearing a complete lack of O-antigen (data not shown). This is likely due to the fact that the structures of the O-antigen and core oligosaccharides are quite different, compared to the structural differences between the full-length and truncated $\mathrm{O}$-antigen (Lam et al., 2011). Thus, although phage mutants can adapt

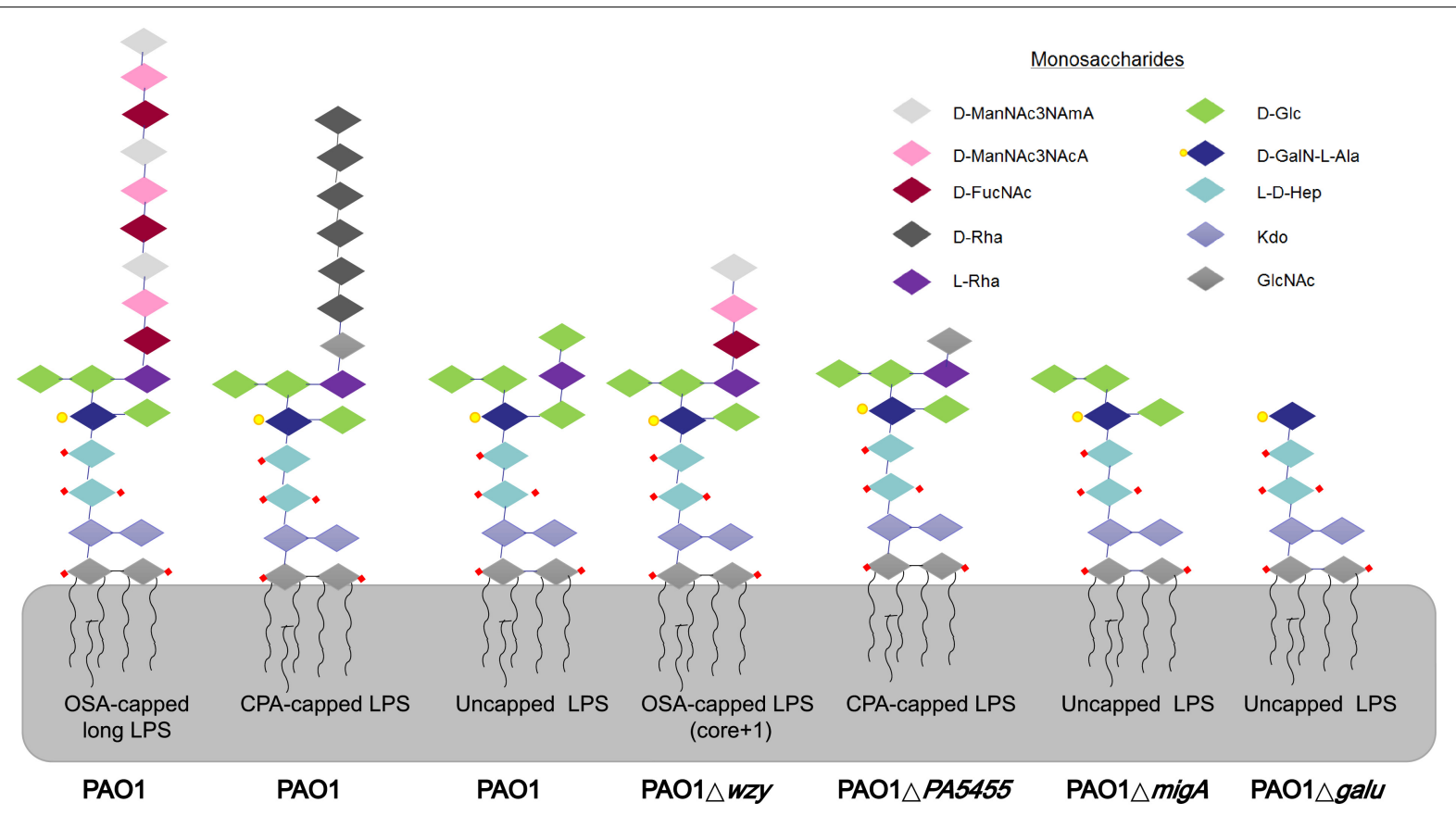

FIGURE 2 | LPS glycoforms on the surface of $P$. aeruginosa strains. Wild-type PAO1 forms OSA-capped LPS, CPA-capped, and uncapped LPS. PAO1 $\triangle$ Wzy has only one copy of the typically repeating O-antigen sugar unit. PAO1 $\triangle$ gall $U$ has no O-antigen, exposing the uncapped core. PAO1 $\triangle P A 5455$ has only GlcNAc, instead of the full-length CPA. PAO1 $\triangle$ migA lacks the L-Rha and D-Glc normally observed on the uncapped core. d-ManNAc3NAcA:di-N-acetylated mannuronic acid; D-FucNAc: d-2-amino-2,6-dideoxy-galactose-acetamido; D-Rha: D-rhamnose; L-Rha: L-rhamnose; D-Glc: D-glucose; D-GalN-L-Ala: d-galactosamine-I-alanyl; L-D-Hep: L-glycero-d-manno-heptose; Kdo: 3-deoxy-d-manno-oct-2-ulosonic acid; GlcNAc: glucose-acetamido. 
A

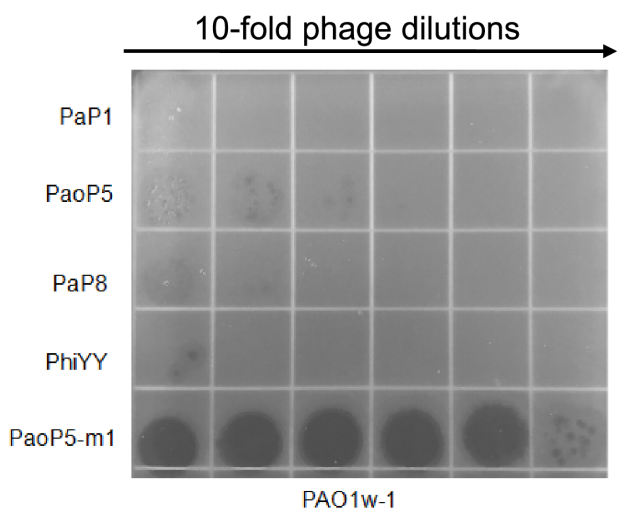

C

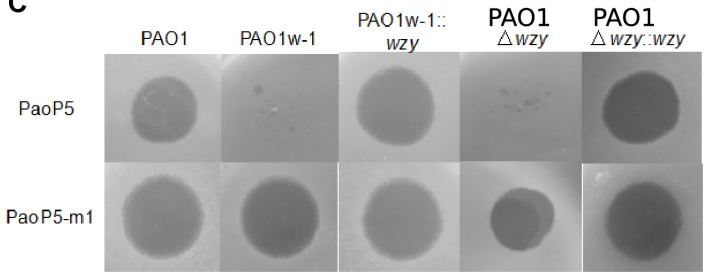

B

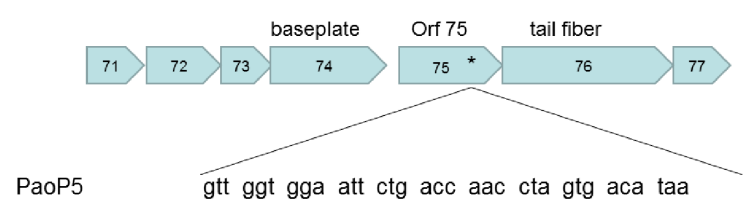

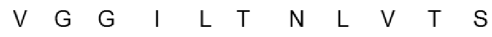

PaoP5-m1

gtt ggt gga att ctg ccc aac cta gtg aca taa

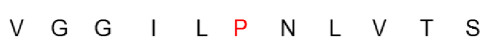

PaoP5-m2 gtt ggt gga att ctg ccc aac cta gtg aca taa

PaoP5-m3 gtt ggt gga att ctg ccc aac cta gtg aca taa

PaoP5-m4 gtt ggt gga att ctg ccc aac cta gtg aca taa

PaoP5-m5 gtt ggt gga att ctg ccc aac cta gtg aca taa

D

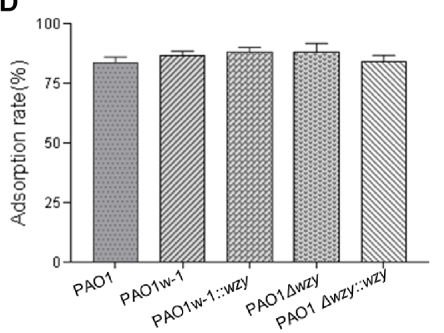

E

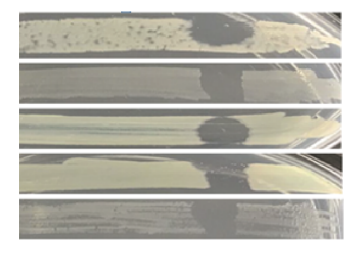

FIGURE 3 | Phage PaoP5-m1 is able to infect $P$. aeruginosa phage-resistant mutants with truncated O-antigen structures. (A) Phage spot assay that indicates PaoP5-m1, but not PaP1, PaoP5, PaP8, and phiYY, efficiently infects PAO1w-1. (B) PaoP5-m1-5 contain an A715C mutation in orf75. PaoP5-m1 efficiently infects (C) and adsorbs (D) to all the PAO1 derived strains with full or truncated O-antigen structure. (E) Phage spot assay indicating five additional $P$. aeruginosa mutants selected by resistance to dsDNA phage PaoP5 are sensitive to PaoP-m1.

rapidly to a truncated receptor structure, they cannot adapt to a completely novel receptor structure in such a short period of time (Schwartz and Lindell, 2017).

\section{Generation of a Five-Phage Cocktail With Broad Host Range}

As the genotypes and phenotypes exemplified by PAO1r-1 and PAO1w-1 represented the two major types of resistant mutants which emerged following dsDNA phage treatment of $P$. aeruginosa, phiYY and PaoP5-m1 were included in a 5-phage cocktail we formulated to constrain the emergence of resistance during phage therapy of $P$. aeruginosa. In addition to phiYY and PaoP5-m1, the parent phage of PaoP5-m1, PaoP5, and 2 additional dsDNA phages with a wide host range ( $\mathrm{PaP} 1$ and $\mathrm{PaP} 8$ ) were included in the phage cocktail. The effectiveness of this cocktail at killing $P$. aeruginosa was examined against a panel of strains that included the type strains PAO1 and PA1, the phage-resistant mutants PAO1-r-1 and PAO1-w-1, as well as 19 $P$. aeruginosa clinical isolates from seven Chinese hospitals (Yang et al., 2016). To maximize the diversity of the panel, the 19 clinical isolates were selected based on diverse Eric-PCR typing (Khosravi et al., 2016; Yang et al., 2016): two strains were selected from clusters 1, 4, and 5, while one strain was selected from each of the remaining 13 typing clusters (Figure 4).

The efficiency of plating (EOP) of each phage and the 5phage cocktail was measured across the panel of $23 P$. aeruginosa strains (Figure 4). EOP is more accurate than a simple dot plaque assay in determining phage sensitivity and host range, as the phage lysate is serially diluted to minimize the impact of other antimicrobial agents, such as lysin, within the lysate (Forti et al., 2018). Interestingly, phiYY was able to kill 12 of the strains on the panel, however the EOP was low. Each phage had a distinct host range, and no individual phage lysed all strains in the panel. As expected, the 5-phage cocktail had by far the widest host range and high EOP values.

\section{The Phage Cocktail Inhibits Bacterial Growth in Liquid Cultures and in Biofilms}

To test the effects of the individual phages or the phage cocktail on a liquid culture of $P$. aeruginosa, and provide more detail regarding the lysis kinetics of each treatment, cultures of PAO1 or PA1 were infected with each phage or the cocktail, and the optical density $\left(\mathrm{OD}_{600}\right)$ was monitored over $5 \mathrm{~h}$ (Figure 5A). For PA1, which is sensitive to $\mathrm{PaP} 1$, the addition of $\mathrm{PaP} 1$ resulted in a decrease in the $\mathrm{OD}_{600}$ of PA1 within $1 \mathrm{~h}$ after infection. Meanwhile, challenge with PaP8 did not significantly affect $\mathrm{OD}_{600}$ compared to the no phage negative control, which is likely due to the low infection efficiency of PaP8 against PA1, as seen above in Figure 4. In PAO1, phage PaoP5 infection caused a decrease in $\mathrm{OD}_{600}$ 1-2 h post-infection, and PaP8 could kill and inhibit PAO1 growth, as indicated by the relatively stable $\mathrm{OD}_{600}$. When treated with the 5-phage cocktail, the $\mathrm{OD}_{600}$ of both the PAO1 and PA1 cultures decreased about $1 \mathrm{~h}$ after phage infection, indicating that the phage cocktail was effective in lysing liquid 
TABLE 2 | Primers used in this study.

\begin{tabular}{|c|c|}
\hline Primers & Sequence $\left(5^{\prime}-3^{\prime}\right)$ \\
\hline \multicolumn{2}{|l|}{ Validating wzy mutation } \\
\hline Wzy-F1 & TCACTGAAACAGGTCGGTAT \\
\hline Wzy-R1 & TACCTATAACAACAGCAATCG \\
\hline \multicolumn{2}{|l|}{ Knockout of wzy } \\
\hline Wzy-K-F(EcoRl) & gGAATTCCTCGCCGTGCACTTGCTCGT \\
\hline Wzy-K-R(BamHI) & cgGGATCCGCCAAACACCTCATGTTCCA \\
\hline \multicolumn{2}{|l|}{ Complementation of wzy } \\
\hline Wzy -C-F(Kpnl) & ggGGTACCCTTGCCGTCACTITCTCCGA \\
\hline Wzy -C-R(Pstl) & aaCTGCAGGGAGTTGGCGCATATGCATA \\
\hline \multicolumn{2}{|l|}{ Complementation of galU } \\
\hline galU -C-F(BamHI) & $\begin{array}{l}\text { cgGGATCCTATGATCAAGAAATGTC } \\
\text { गITCCCG }\end{array}$ \\
\hline galU -C-R(Pstl) & $\begin{array}{l}\text { aaCTGCAGTCAGTGAGCCTTGCC } \\
\text { GGTCTTGT }\end{array}$ \\
\hline \multicolumn{2}{|l|}{ Mapping mutations in PaoP5-m1 } \\
\hline 062orf-F1 (structural protein-F1) & TITGGAAGACCTACCCCTC \\
\hline 062orf-R1 (structural protein-R1) & GCCTTCCTGAACAACACTGA \\
\hline 063orf-F1 & GACATGGCAATGGCGATTAC \\
\hline 063orf-R1 & GTCGTAGTTGTAAAGAGCAG \\
\hline 064orf-067orf-F1 & CGTCTGTGGTTCCGCATGGC \\
\hline 064orf-067orf-R1 & AATITAAGTTCCCCAGTAAG \\
\hline $\begin{array}{l}\text { 068orf-F1 (tape measure } \\
\text { protein-F1) }\end{array}$ & CGGTIIITCTCTAATCGGT \\
\hline $\begin{array}{l}\text { 068orf-R1 (tape measure } \\
\text { protein-R1) }\end{array}$ & CAAATCTAGACGTTGTGATAACA \\
\hline 069orf-073orf-F1 & TAGCGACAGACTTCTAGCTC \\
\hline 069orf-073orf-R1 & CTGGTAGGGCGAGCCGGATA \\
\hline 074orf-F1 & TGGTAGACATTGTTGCTCAA \\
\hline 074orf-R1 & TTCCAGCGAATCCAAAATAG \\
\hline 075orf-F1 & ATAGAGATCAAGATCAGTGTAACA \\
\hline 075orf-R1 & TCGCTACCTGTATGGGTACG \\
\hline 076orf-F1 (tailfiber1-F1) & TCGAAGCTTATAAATITGTATTCG \\
\hline 076orf-R1 (tailfiber1-R1) & TCGATAGCAATCTCTACTTGACG \\
\hline 077orf-F1 & AAACTGACGTAATGACTGATGC \\
\hline 077orf-R1 & GGAATCTTGTCGGAATGGGT \\
\hline 078orf-F1 (tailfiber2-F1) & TAGAGGGCTGTCCCACATCT \\
\hline 078orf-R1 (tailfiber2-R1) & CAGTCTCTGTCCATGTTGACTG \\
\hline 079orf-F1 (endolysin-F1) & GCCAATCGGATATITCCAGA \\
\hline 079orf-R1 (endolysin-R1) & TTGCGGCAGCTACTACGAG \\
\hline 080-088orf-F1 & AGACTGCGCCTTGCAAAG \\
\hline 080-088orf-R1 & CAAGCCAGAATACTACGTCTATGA \\
\hline
\end{tabular}

cultures of both $P$. aeruginosa strains, and it did so in a similar timeframe as the challenge with the single, effective phages.

The capability of phages to reduce biofilms formed by $P$. aeruginosa PA1 or PAO1 was examined using crystal violet staining. Following $24 \mathrm{~h}$ of biofilm formation by $P$. aeruginosa strains on 96-well plates, phages were applied individually or in the 5-phage cocktail. Plates were incubated for $4 \mathrm{~h}$, at which point the biofilms were stained with crystal violet. Challenge with the phage cocktail caused a significant reduction in the biofilm biomass of both PA1 and PAO1 (Figure 5B), and eliminated biofilms with a slightly higher efficacy compared to that of challenge using the single, effective phage.

\section{The Phage Cocktail Inhibits the Development of Phage-Resistant Bacteria in the Short-Term}

To determine whether the 5-phage cocktail inhibits the emergence of phage-resistant mutants, $P$. aeruginosa PAO1 or PA1 was mixed with a single phage or the phage cocktail. When treated with a single phage, the $\mathrm{OD}_{600}$ of PAO1 and PA1 suffered an initial decrease during the first few hours. However, both strains re-grew to high densities after $12-24 \mathrm{~h}$ of incubation, most likely due to the growth of phage-resistant mutants. On the other hand, when treated with the 5-phage cocktail, the $\mathrm{OD}_{600}$ of both strains remained low even after $48 \mathrm{~h}$ of incubation (Figure 6). This data strongly indicates that the 5-phage cocktail not only efficiently kills $P$. aeruginosa, but also prevents the emergence of phage-resistant mutants in vitro.

\section{After Extended Exposure to the Phage Cocktail, Resistant Isolates Emerge With Mutations in migA and wzy}

To test whether resistance to the 5-phage cocktail emerged after a longer period of time, the $P$. aeruginosa PAO1 culture containing the phage cocktail was incubated for an additional 7 days without the addition of fresh growth media (Figure 7A). In all three replicates, bacterial density finally began to increase after around 5 days $\left(\mathrm{OD}_{600}=0.5 \sim 1.1\right)$, indicating that resistance to the phage cocktail had developed. These cultures were plated on LB agar and two colonies were isolated from each plate (Table 3 ). The EOP of each selected mutant was examined, and all six isolates were indeed resistant to all phages in the cocktail as no plaques were formed. To determine a genetic cause of the phage resistance, these six isolates, and wild type PAO1, were subjected to whole genome sequencing. The sequencing data revealed that all 6 isolates contained mutations in both wzy and migA (Table 3).

Many $P$. aeruginosa simultaneously synthesize multiple forms of lipopolysaccharide (Figure 2). These include the capped core glycoforms O-antigen-specific (OSA) and common polysaccharide antigen (CPA), as well as the uncapped core. The enzyme encoded by migA is responsible for the transfer of an L-rhamnose to glucose II of the core polysaccharide, and is required for the synthesis of uncapped core. Thus, the migA mutant lacks the L-rhamnose residue on the uncapped cores (Kocincova et al., 2012), and the wzy mutant lacks the OSA and produces "core + 1" LPS (Li et al., 2018). The sequencing data presented here implies that mutations in both wzy and migA are required for phage cocktail resistance. As expected, although both PAO1 $\Delta w z y$ and PAO1 $\Delta m i g A$ were sensitive to PaoP5-m1, a PAO1 $\Delta w z y \Delta m i g A$ double mutant was resistant to PaoP5-m1 (Figure 7B). This data implies that PaoP5-m1 may use either core antigen or OSA as receptors.

Among the six selected isolates resistant to the 5-phage cocktail, four had additional mutations. These mutations were located in the genes gmd, rmd or PA5455 (Table 3), all of three of which are involved in CPA synthesis (Lam et al., 2011; Hao et al., 2013). These mutations are less likely to be involved in the resistance to the phage cocktail seen in these strains, as 


\begin{tabular}{|c|c|c|c|c|c|c|c|c|}
\hline \multirow{2}{*}{$\begin{array}{l}\text { Bacterial } \\
\text { Strain }\end{array}$} & \multicolumn{6}{|c|}{ Efficiency of Plating of } & \multirow{2}{*}{$\begin{array}{l}\text { Isolated } \\
\text { From }\end{array}$} & \multirow{2}{*}{$\begin{array}{c}\text { ERIC-PCR } \\
\text { Cluster }\end{array}$} \\
\hline & PaP1 & PaoP5 & PaP8 & phiYy & PaoP5-m1 & Cocktail & & \\
\hline PAO1 & - & + & + & - & + & + & -- & 5 \\
\hline PAO1r-1 & - & - & - & + & - & + & -- & 5 \\
\hline PAO1w-1 & - & - & - & - & + & + & -- & 5 \\
\hline PA1 & + & - & $-1+$ & - & + & + & $X Q$ & 1 \\
\hline L14 & + & + & + & $-1+$ & + & + & $\mathrm{HN}$ & 2 \\
\hline $\mathrm{J} 15$ & + & + & + & $+/-$ & $-1+$ & + & $\mathrm{XJ}$ & 12 \\
\hline $\mathrm{X} 12$ & $-1+$ & $+/-$ & $-1+$ & $+/-$ & $+1-$ & $+/-$ & $X Q$ & 4 \\
\hline $\mathrm{N} 1$ & - & + & $+/-$ & - & + & + & SW & 10 \\
\hline S18 & - & - & $+/-$ & - & - & $-1+$ & SC & 4 \\
\hline H15 & $-1+$ & - & $-1+$ & - & - & - & WC & 5 \\
\hline N13 & - & - & $+/-$ & $+1-$ & - & + & SW & 6 \\
\hline N65 & $-1+$ & $+/-$ & $-1+$ & $-1+$ & $+/-$ & $+/-$ & SW & 7 \\
\hline N30 & + & + & + & $-1+$ & + & + & SW & 13 \\
\hline L67 & - & - & - & - & - & - & $\mathrm{HN}$ & 8 \\
\hline X9 & + & + & $-1+$ & $+1-$ & + & + & $X Q$ & 16 \\
\hline N32 & - & - & - & $+1-$ & - & $+/-$ & SW & 11 \\
\hline L75 & + & + & + & $-1+$ & + & + & $\mathrm{HN}$ & 14 \\
\hline $\mathrm{H} 1$ & $-1+$ & $-1+$ & + & $-1+$ & $+/-$ & + & WC & 5 \\
\hline N22 & - & + & $-1+$ & - & + & + & SW & 1 \\
\hline $\mathrm{X} 8$ & $-1+$ & + & + & $-1+$ & + & + & $X Q$ & 15 \\
\hline $\mathrm{H} 19$ & - & - & + & - & - & + & WC & 1 \\
\hline N42 & - & + & $-1+$ & - & + & + & SW & 3 \\
\hline D9 & + & - & $+/-$ & - & - & + & DP & 9 \\
\hline
\end{tabular}

FIGURE 4 | EOP of single phages or a 5-phage cocktail on a panel of $23 P$. aeruginosa strains. The killing efficiency of each phage strain and cocktail were determined by an EOP assay. $(+)=$ EOP $1 ;( \pm)=$ EOP $0.1-0.01 ;(-/+)=$ EOP $0.001 ;(-)=$ EOP $<0.0001$. For clinical isolates of $P$. aeruginosa, the "Isolated From" column lists the hospital where the strain was isolated: SW, SouthWest Hospital; DP, DaPing Hospital; XQ, XinQiao Hospital; WC, West China Hospital; SC, SiChuan Provincial People's Hospital; XJ, XiJing Hospital; HN, HeNan Provincial People's Hospital.

the PAO1 $\Delta w z y \Delta$ migA double mutation was sufficient to confer resistance to the phage cocktail.

\section{DISCUSSION}

Pseudomonas aeruginosa is an opportunistic pathogen which poses a serious health threat, particularly to patients with cystic fibrosis or traumatic burns, as well as the immunocompromised. $P$. aeruginosa is a master of antibiotic resistance, with intrinsic resistance to several drugs via low membrane permeability and expression of efflux pumps, and has a remarkable capacity to mutate and horizontally acquire additional traits leading to multidrug-resistant (MDR) and extensively-resistant (XDR) strains (Lopez-Causape et al., 2018). With the emergence of these phenotypes, which severely limit current treatment options, phage therapy is once again being explored as a potential therapeutic. However, phage therapy is still not widely used due to legislation issues and the lack of proper clinical trials and efficacy studies. As to the phage agents, one of the major barriers to successful phage therapy is the rapid emergence of phage-resistant mutants (Rohde et al., 2018).

Co-evolution of phages and their hosts is common in the natural environment, and the mutation and modification of receptors and receptor binding proteins by both phage and their hosts is well-understood as a major mechanism for this classic Red Queen phenomenon (Paterson et al., 2010). Indeed, one of the major mechanisms of phage resistance in $P$. aeruginosa is modifications to the LPS molecules exposed on the cell surface. As mutant phages with an expanded or altered host range can be isolated through phage training, and serve as complementary components of a therapeutic phage cocktail to prevent emergence of phage-resistant mutants, the goal of this study was to further characterize phage-resistant mutants of $P$. aeruginosa, perform phage training to isolate phages that break these resistant phenotypes, and develop a phage cocktail effective at constraining the development of phage-resistant $P$. aeruginosa during treatment. 

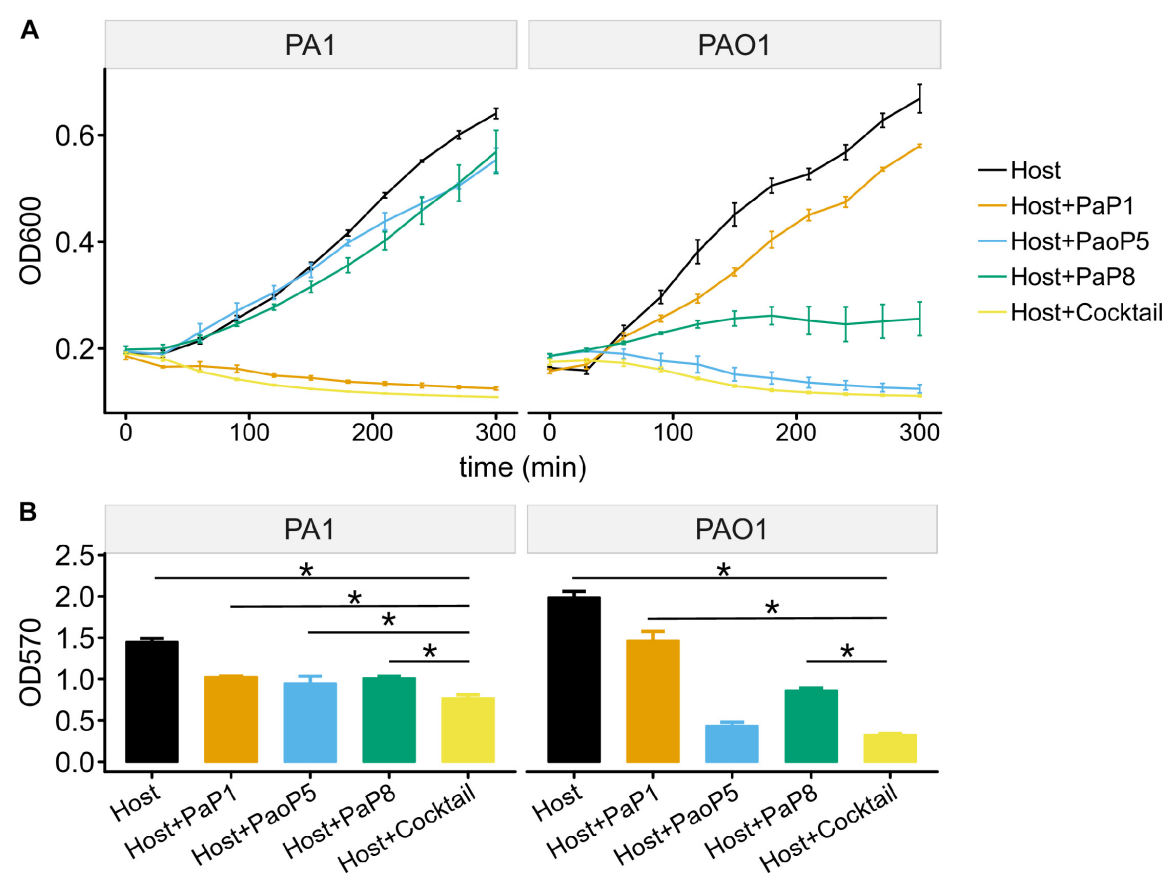

FIGURE 5 | Effects of the 5-phage cocktail on liquid cultures and biofilms of $P$. aeruginosa. (A) Growth kinetics of $P$. aeruginosa cells in liquid culture in presence or absence of single phage strains or the phage cocktail. (B) Disruption of $P$. aeruginosa biofilms by single phage strains or the phage cocktail. ( $P<0.05$, one-way ANOVA, $n=3$ ). The asterisks mark $P$-value of $<0.05$ as calculated by Student's $t$-test between two groups.

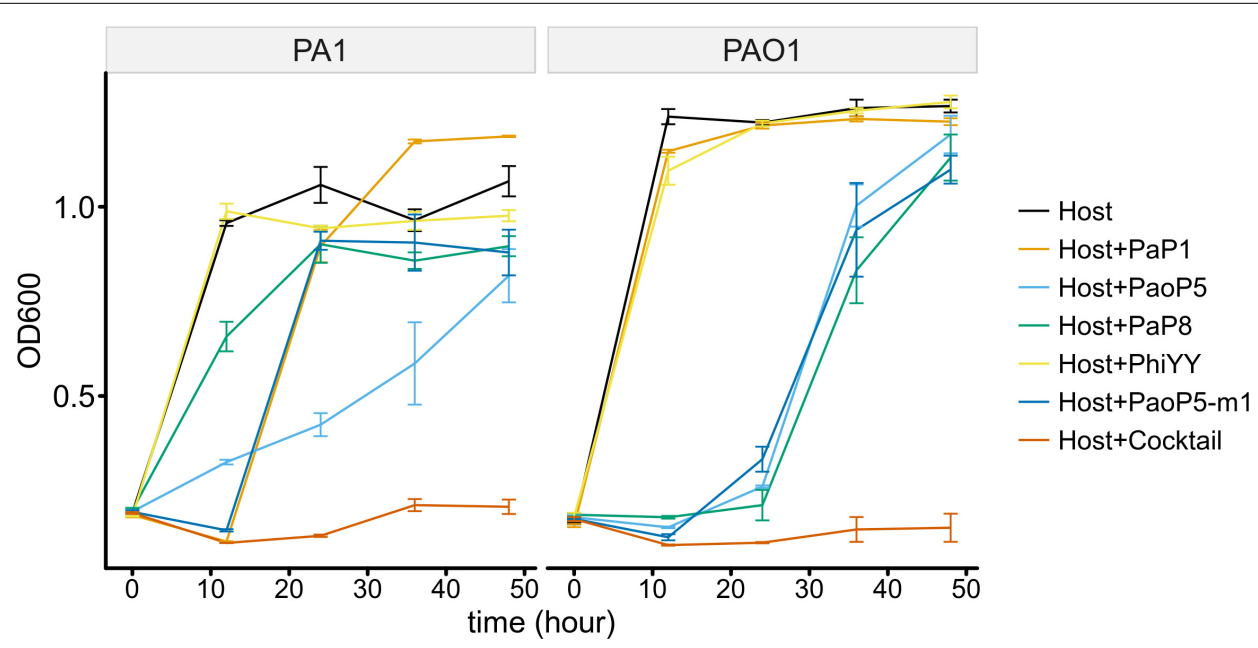

FIGURE 6 | The 5-phage cocktail constrains phage-resistant mutants in the short-term. Growth kinetics of $P$. aeruginosa PA1 and PAO1 in liquid cultures in the presence or absence of single phage strains or the phage cocktail for $48 \mathrm{~h}$.

The mutant strain PAO1r-1, derived from $P$. aeruginosa PAO1, was resistant to dsDNA phages, but is susceptible to infection by the dsRNA phage, phiYY (Figure 1). This is due to the fact that PAO1r-1 contains a large deletion, including galU, which leads to truncated LPS, with core oligosaccharides exposed. phiYY appears to utilize these core oligosaccharides as the receptor for infection (Figure 1). Further credence for this hypothesis was provided by the fact that phiYY did not infect or adsorb the parent strain with a complete LPS, or a the complement strains PAO1r-1::galU or PAO1 1 galU::galU (Figure 1).

dsRNA phages are a unique group of phages, which are classified in the Cystoviridae family (Mantynen et al., 2018). Currently, there are only 7 sequenced dsRNA phages, and phiYY is the only one that infects human pathogen (Yang et al., 2016), while other 6 dsRNA phages all infects Pseudomonas syringae. phi6 is the first isolated dsRNA phage, and serves as an excellent model to study the biology of dsRNA phage (Bamford et al., 

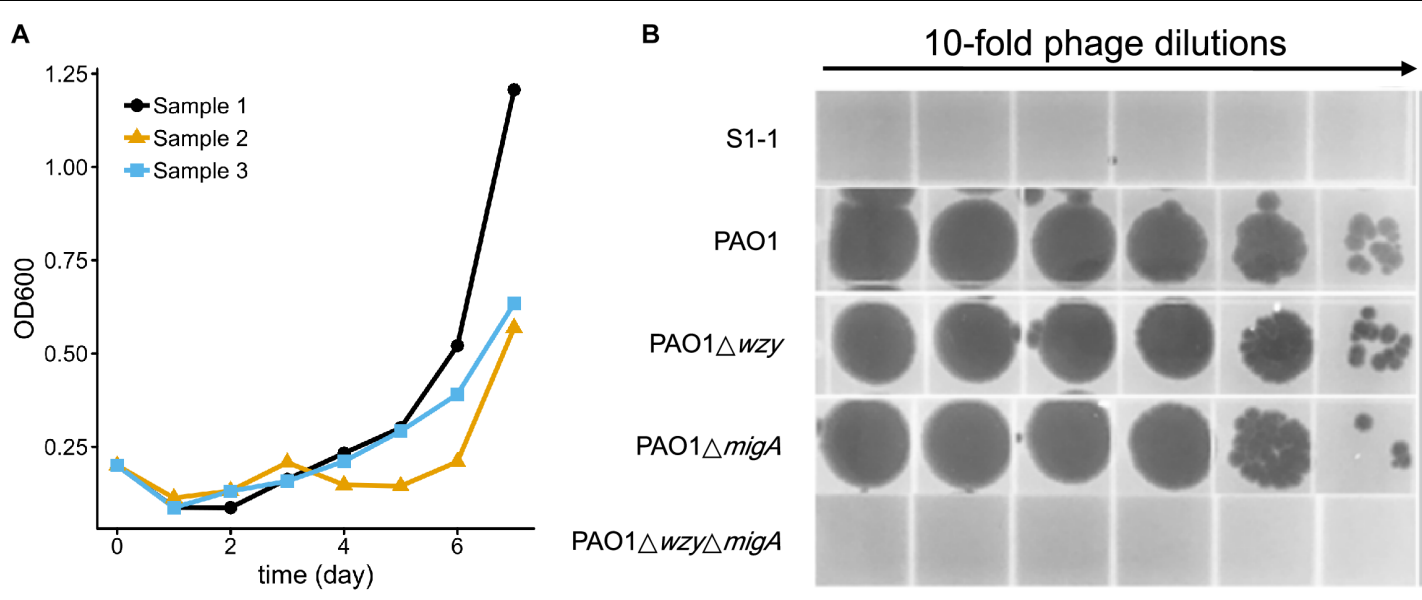

FIGURE 7 | Resistance to the 5-phage cocktail emerges after 7 days, and requires mutations in wzy and migA. (A) Growth kinetics of $P$. aeruginosa PAO1 in liquid culture in the presence of the phage cocktail for 7 days. Three biological repeats were performed. (B) Phage spot assay illustrating that the phage cocktail resistant mutant S1-1 and PAO1 $\Delta$ wzy $\Delta$ migA double mutant are resistant to infection by PaoP5-m1, while single-gene disruptions of PAO1 $\Delta$ wzy and PAO1 $\Delta$ migA are sensitive to PaoP5-m1.

TABLE 3 | Mutations found in phage cocktail resistant mutants.

\begin{tabular}{|c|c|c|c|c|c|}
\hline Sample & Nucleotide change & Amino acid change & Affected gene & Affected protein & Function \\
\hline \multirow[t]{2}{*}{$S 1-1 / S 1-2$} & $\mathrm{~T} \rightarrow \mathrm{G}$ & $\mathrm{H} 123 \mathrm{P}$ & $\operatorname{migA}$ & alpha-1,6-rhamnosyltransferase & Uncapped core \\
\hline & $(T)_{7 \rightarrow 9}$ & Shift (130/1317 nt) & $w z y$ & B-band O-antigen polymerase & OSA \\
\hline \multirow[t]{4}{*}{$S 2-1$} & $\mathrm{G} \rightarrow \mathrm{A}$ & Q42* & $\operatorname{migA}$ & alpha-1,6-rhamnosyltransferase & Uncapped core \\
\hline & $(\mathrm{CTGGCC})_{2 \rightarrow 3}$ & coding (1353/1689 nt) & fimL & hypothetical protein & Unkonwn \\
\hline & $\mathrm{G} \rightarrow \mathrm{A}$ & P73S & $w z y$ & B-band O-antigen polymerase & OSA \\
\hline & $(\mathrm{CCA})_{2 \rightarrow 3}$ & coding (95/972 nt) & gmd & GDP-mannose 4,6-dehydratase & CPA \\
\hline \multirow[t]{4}{*}{ S2-2 } & $\mathrm{G} \rightarrow \mathrm{A}$ & Q42* & migA & Alpha-1,6-rhamnosyltransferase & Uncapped core \\
\hline & $(\mathrm{CTGGCC})_{2 \rightarrow 3}$ & coding (1353/1689 nt) & fimL & hypothetical protein & Unkonwn \\
\hline & $\mathrm{G} \rightarrow \mathrm{A}$ & P73S & wzy & B-band O-antigen polymerase & OSA \\
\hline & $\mathrm{C} \rightarrow \mathrm{A}$ & G31C & gmd & GDP-mannose 4,6-dehydratase & CPA \\
\hline \multirow[t]{3}{*}{ S3-1 } & $\mathrm{C} \rightarrow \mathrm{T}$ & $\mathrm{R} 217 \mathrm{Q}$ & migA & Alpha-1,6-rhamnosyltransferase & Uncapped core \\
\hline & $\mathrm{G} \rightarrow \mathrm{A}$ & P73S & wzy & B-band O-antigen polymerase & OSA \\
\hline & $\mathrm{C} \rightarrow \mathrm{T}$ & W151* & rmd & oxidoreductase Rmd & CPA \\
\hline \multirow[t]{3}{*}{ S3-2 } & $\mathrm{C} \rightarrow \mathrm{T}$ & $\mathrm{R} 217 \mathrm{Q}$ & migA & Alpha-1,6-rhamnosyltransferase & Uncapped core \\
\hline & $\mathrm{G} \rightarrow \mathrm{A}$ & P73S & wzy & B-band O-antigen polymerase & OSA \\
\hline & $\mathrm{G} \rightarrow \mathrm{A}$ & W100* & PA5455 & hypothetical protein & CPA \\
\hline
\end{tabular}

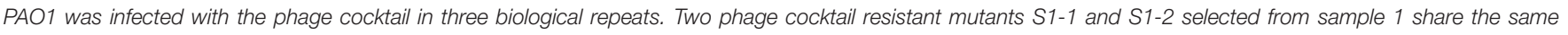

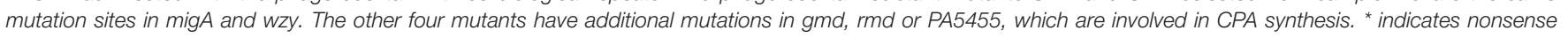
mutation.

1987), while our study demonstrated the antimicrobial potential of dsRNA phage phiYY, which eliminates the brown mutants after infection with dsDNA phages. Moreover, brown mutants are also frequently detected from cystic fibrosis patients with chronic $P$. aeruginosa infections, suggesting the brown mutants can better survive in chronic infection (Smith et al., 2006; Hocquet et al., 2016). Thus, dsRNA phage phiYY might be an efficient agent to kill the brown mutants in chronic $P$. aeruginosa infections, which needs further investigation.

Meanwhile, mutations in another locus involved in LPS production, $w z y$, generated phage-resistant mutants in $P$. aeruginosa PA1, which express a truncated "core +1 " LPS with only one repeat of the O-antigen trisaccharide moiety. Similar phage-resistant wzy mutants were obtained in this study from a PAO1 genetic background (Figure 3). Phage training of PaoP5 generated mutant phages capable of breaking the resistance afforded to PAO1 by the wzy mutation harbored by PAO1w-1, including the phage isolate, PaoP5-m1 (Figure 3). PaoP5-m1 contains a mutation in orf75, and was capable of adsorbing to and infecting PAO1 and all tested $w z y$ mutants that arose following infection of PAO1 with PaoP5 (Figure 3). Phage-host co-evolution could expand the host range of the phages and increase the resistance of evolved bacteria (Paterson et al., 2010). Thus, mutant phages with expanded or altered host range can be isolated through phage training, and serve as auxiliary 
components of a phage cocktail to eliminate the phage resistant bacteria. However, a similar phage training approach failed to produce phage that could break the resistance phenotype of PAO1r-1, likely because the exposed LPS core oligosaccharides of PAO1r-1 are too structurally disparate from the full-length LPS.

A 5-phage cocktail was generated containing three $P$. aeruginosa phages with a broad host range (PaP1, PaoP5, PaP8), as well as the two phages capable of breaking P. aeruginosa phage resistance mechanism described above (phiYY and PaoP5-m1). This phage cocktail was examined against a panel of $P$. aeruginosa strains including two laboratory type strains relevant to this study (PAO1 and PA1), the phage-resistant mutants described above (PAO1r-1 and PAO1w-1), and 19 clinical isolates which were selected to represent the 15 EricPCR typing clusters. The phage cocktail was able to infect and kill $\sim 90 \%$ of the strains tested, higher than each of the components of the cocktail individually, and did so with increased EOP values (Figure 4). The phage cocktail was effective in killing $P$. aeruginosa in liquid cultures and biofilms, in addition to solid media (Figure 5). While the phage cocktail was effective in constraining the emergence of phage-resistant mutants during the time frames utilized to develop the phageresistant mutants described above, extended incubation out to 5-7 days led to growth of mutants resistant to the cocktail. All isolated mutants which were resistant to the cocktail had mutations in both $w z y$ and migA. This development of resistance requiring mutations in multiple loci is a classic illustration of a Red Queen dynamic.

In the natural environment, phage-host co-evolution dynamics have been described as arms race fluctuation selection. It is intriguing to see that this study, while the inclusion of phiYY and PaoP5-m1, the two phages that target two main types of phage resistant mutants in $P$. aeruginosa, significantly constrained the emergence of phage resistant mutants, mutants carrying double or triple gene mutations, including $w z y$ and migA eventually appeared after 5 days' incubation. Thus, a better understanding of the mechanism of how these mutants evolved even after long term incubation is important in designing better phage-therapy strategy, and warrant further investigation.

\section{EXPERIMENTAL PROCEDURES}

\section{Bacterial Strains, Phages and Culture Conditions}

The bacterial strains and phages in this work are listed in Table 1. $P$. aeruginosa strains were grown on Luria-Bertani (LB) broth at $37^{\circ} \mathrm{C}$. When required, gentamicin $(50 \mu \mathrm{g} / \mathrm{ml})$, or tetracycline $(35 \mu \mathrm{g} / \mathrm{ml})$ were used at a final concentrations in LB broth as described below. To completely remove bacteria, dsDNA phages were treated with chloroform, while dsRNA phage was filtered multiple times through $0.22 \mu \mathrm{m}$ filters (Millipore), because phiYY is chloroform sensitive. To generate the phage cocktail, 5-phages with the same titer were mixed to a final titer of $10^{9} \mathrm{pfu} / \mathrm{ml}$.

\section{Selection of Phage-Resistant Mutants}

The process of isolating phage-resistant mutants and calculating the frequency of resistance has been described in detail previously (Shen et al., 2018). PAO1r-1 was previously isolated, tested and sequenced (Shen et al., 2018). To isolate a PAO1-derived whitephenotype mutant PAO1w-1, PAO1 was infected with PaoP5 and immediately plated on LB agar plates for $24 \mathrm{~h}$. Then, the white mutants were isolated. The $w z y$ gene was PCR amplified with primers listed in Table 2, and the PCR products were sequenced to identify the mutation site in $w z y$.

\section{Construction of $P$ aeruginosa Strains}

The knockout and complementation of galU and wzy were performed as previously described (Le et al., 2014; Li et al., 2018). Briefly, to complement galU in PAO1r-1 and PAO1 $\Delta$ galU, the galU gene was PCR amplified using primers galU-C-F and galU-C-R, and the PCR product was digested by BamHI/PstI, and cloned into the BamHI/PstI digested plasmid pUCP24 to generate pucp-galU. Then, pucp-galU was electroporated into PAO1r-1 or PAO1 $\Delta$ galU. The sample approach was applied to complement $w z y$ in $\mathrm{PAO} 1 \mathrm{w}-1$ and PAO $1 \Delta w z y$ with primers Wzy-C-F and Wzy-C-R.

To make insertional deletion of $w z y$ in PAO1 and PAO1 $\Delta m i g A$, a fragment within $w z y$ was amplified with primers $\mathrm{Wzy}-\mathrm{K}-\mathrm{F}$ and $\mathrm{Wzy}-\mathrm{K}-\mathrm{R}$, and the PCR product was digested by BamHI/EcoRI, and cloned into the BamHI/EcoRI digested plasmid pEX18Gm to generate plasmid pEX-wzy. Then, pEX-wzy was electroporated into PAO1 or PAO1 1 migA to generate insertional mutant.

\section{Bacteriophage Adsorption Assay}

A bacteriophage adsorption assay with various $P$. aeruginosa strains was performed according to a previously described protocol (Le et al., 2014). Briefly, the log phase bacterial cultures were harvested and resuspended in LB broth with an $\mathrm{OD}_{600}$ of 0.5 . Phage was then added at an MOI of 0.01 , and the adsorption proceeded at $37^{\circ} \mathrm{C}$ for $5 \mathrm{~min}$. Then, $1 \mathrm{ml}$ samples were collected and centrifuged at $16,000 \times g$ for $1 \mathrm{~min}$. The supernatant was filtrated using $0.22-\mu \mathrm{m}$-pore-size filters (Millipore). Then, the phage titer in the supernatant $(\mathrm{t} 1)$ and the original phage stock (t0) were determined using double-agar plating assays. The phage adsorption rate was calculated as $(\mathrm{t} 0-\mathrm{t} 1) / \mathrm{t} 0$. The values of adsorption rate shown in the bar graph are the means and standard deviations are the error bars from three biological replicates.

\section{EOP Assay and Phage Spot Assay}

The EOP of each phage isolate and the 5-phage cocktail on $P$. aeruginosa strains was determined according to a previously described protocol (Forti et al., 2018). $5 \mu$ l of serial 10-fold dilutions of phage were spotted on double layer agar plates, on which a specific bacterial host was spread in the upper soft agar. The number of plaques observed after overnight incubation were compared to the number obtained on sensitive strain PAO1, PA1 or PAO1r-1. The phage spot assay is similar to the EOP assay. Mix $200 \mu \mathrm{l}$ of bacterial culture with $4 \mathrm{ml}$ of soft agar and pour in the 
agar plate. Then drop $1 \mu$ l of phage on the soft agar and observe the formation of plaque after overnight incubation.

\section{Isolation and Sequencing of Mutant PaoP5 Phages}

The process of phage training to break resistance has been described previously (Le et al., 2013). Briefly, $\sim 10^{7}$ pfu of phage PaoP5 was mixed with $200 \mu \mathrm{l}$ of log phase $P$. aeruginosa strain PAO1 $\left(\mathrm{OD}_{600}=0.5\right)$ and plated in a double-layer plate. Dozens of plaques appeared after overnight culture. Five clear plaques were randomly selected and transferred into the LB medium. After serial 10- fold dilutions, the plaque assay was repeated with $10 \mu \mathrm{l}$ of each dilution to obtain well-separated plaques. The same procedure was repeated twice to purify each mutant phage. After purification of mutant phage, genomic DNA was extracted as previously described (Le et al., 2013). Subsequently, the whole region encoding the structural proteins (Orf62 to Orf 88) was amplified by PCR using the primers indicated in Table 2. PCR amplicons were then purified and sequenced using Sanger technology. The mutation site was determined by blast the sequencing data with PaoP5 genome (GeneBank accession number KU297675).

\section{Biofilm Disruption Experiment}

Crystal violet staining was used to monitor biofilm disruption (Forti et al., 2018). An overnight culture of $P$. aeruginosa was diluted to $0.02 \mathrm{OD}_{600}$ in $\mathrm{LB}$ broth in 96 -well polystyrene microtiter plates, which were then incubated at $37^{\circ} \mathrm{C}$ for $24 \mathrm{~h}$. The broth was removed and the wells were washed with $200 \mu \mathrm{l}$ of LB. Then, $200 \mu \mathrm{l}$ of LB or LB containing phage or phage cocktail was added, and incubated for $4 \mathrm{~h}$ at $37^{\circ} \mathrm{C}$. Then, crystal violet staining was applied as previously described (Forti et al., 2018). Three biological replicates were performed.

\section{Selection of Mutants Resistant to the Phage Cocktail}

$100 \mu \mathrm{l}$ of phage cocktail was added into $5 \mathrm{ml}$ exponential phase PAO1 $\left(\mathrm{OD}_{600}=0.2\right)$. The culture was incubated at $37^{\circ} \mathrm{C}$ for 7 days with shaking, and the $\mathrm{OD}_{600}$ was monitored.

\section{REFERENCES}

Bamford, D. H., Romantschuk, M., and Somerharju, P. J. (1987). Membrane fusion in prokaryotes: bacteriophage phi 6 membrane fuses with the Pseudomonas syringae outer membrane. The EMBO J.6, 1467-1473. doi: 10.1002/j.14602075.1987.tb02388.x

Barrick, J. E., Colburn, G., Deatherage, D. E., Traverse, C. C., Strand, M. D., and Borges, J. J. (2014). Identifying structural variation in haploid microbial genomes from short-read resequencing data using breseq. BMC Genomics 15:1039. doi: 10.1186/1471-2164-15-1039

Boucher, H. W., Talbot, G. H., Bradley, J. S., Edwards, J. E., Gilbert, D., and Rice, L. B. (2009). Bad bugs, no drugs: no ESKAPE! an update from the infectious diseases society of America. Clin. Infect. Dis. 48, 1-12. doi: 10.1086/595011

Chen, S., Zhou, Y., Chen, Y., and Gu, J. (2018). fastp: an ultra-fast all-in-one FASTQ preprocessor. Bioinformatics 34, i884-i890. doi: 10.1093/bioinformatics/bty560

Choudhury, B., Carlson, R. W., and Goldberg, J. B. (2005). The structure of the lipopolysaccharide from a galU mutant of Pseudomonas aeruginosa serogroupO11. Carbohyd.Res.340, 2761-2772. doi: 10.1016/j.carres.2005.09.017

\section{Bacterial Genome Sequencing}

Bacterial genomic DNA was extracted from six mutants which were resistant to the phage cocktail and wild type PAO1 using the UNlQ-10 Column Bacterial Genomic DNA Isolation Kit (Sangon Biotec), and then sequenced using an Illumina Hiseq 2500 platform ( $\sim$ Gbp/sample). Fastp (Chen et al., 2018) was used for adapter trimming and quality filtering after demultiplexing the raw reads. Mutations were identified in clean reads using Breseq (Barrick et al., 2014) with PAO1 (GenBank accession: NC_002516.2) as the reference genome. The sequence data is available in the NCBI Sequence Read Archive under SRA accession number PRJNA517283.

\section{Statistical Analysis}

The statistical analysis was performed using One-way ANOVA or student's $t$ test. A $P$ value $<0.05$ was considered as statistically significant.

\section{DATA AVAILABILITY STATEMENT}

Publicly available datasets were analyzed in this study. This data can be found here: NCBI Sequence Read Archive under SRA accession number PRJNA517283.

\section{AUTHOR CONTRIBUTIONS}

SL and FH conceived the study. YY, WS, and QZ performed the experiments. QC, KX, XJ, and JW analyzed the data. SL, XH, and JB wrote the manuscript. All authors read and approved the final manuscript for publication.

\section{FUNDING}

This research was supported by the National Natural Science Foundation of China (NSFC, 31870167 to SL) and by NIH/NIDCR F32-DE026947 (JB).

Forde, A., and Hill, C. (2018). Phages of life - the path to pharma. Br.J.Pharmacol.175, 412-418. doi: 10.1111/bph.14106

Forti, F., Roach, D. R., Cafora, M., Pasini, M. E., Horner, D. S., and Fiscarelli, E. V. (2018). Design of a broad-range bacteriophage cocktail that reduces Pseudomonas aeruginosa biofilms and treats acute infections in two animal models. Antimicrob.Agents Chemother. 62:e2573-17.

Hao, Y., King, J. D., Huszczynski, S., Kocincova, D., and Lam, J. S. (2013). Five new genes are important for common polysaccharide antigen biosynthesis in Pseudomonas aeruginosa. mBio 4, e631-e612.

Harvey, H., Bondy-Denomy, J., Marquis, H., Sztanko, K. M., Davidson, A. R., and Burrows, L. L. (2018). Pseudomonas aeruginosa defends against phages through type IV pilus glycosylation. Nat.Microbiol.3, 47-52. doi: 10.1038/s41564-0170061-y

Hocquet, D., Petitjean, M., Rohmer, L., Valot, B., Kulasekara, H. D., and Bedel, E. (2016). Pyomelanin-producing Pseudomonas aeruginosa selected during chronic infections have a large chromosomal deletion which confers resistance to pyocins. Environ. Microbiol. 18, 3482-3493. doi: 10.1111/1462-2920. 13336 
Hyman, P., and Abedon, S. T. (2010). Bacteriophage host range and bacterial resistance. Adv.Appl.Microbiol.70, 217-248. doi: 10.1016/S00652164(10)70007-1

Jacobs, M. A., Alwood, A., Thaipisuttikul, I., Spencer, D., Haugen, E., and Ernst, S. (2003). Comprehensive transposon mutant library of Pseudomonas aeruginosa. Proc. Natl. Acad. Sci. U.S.A. 100, 14339-14344. doi: 10.1073/pnas.2036282100

Jault, P., Leclerc, T., Jennes, S., Pirnay, J. P., Que, Y. A., and Resch, G. (2018). Efficacy and tolerability of a cocktail of bacteriophages to treat burn wounds infected by Pseudomonas aeruginosa (PhagoBurn): a randomised, controlled, double-blind phase 1/2 trial. LancetInfect.Dis.19, 35-45. doi: 10.1016/s14733099(18)30482-1

Jonckheere, L., Schelstraete, P., Van Simaey, L., Van Braeckel, E., Willekens, J., and Van Daele, S. F. (2018). Establishing the diagnosis of chronic colonization with Pseudomonas aeruginosa of cystic fibrosis patients: comparison of the European consensus criteria with genotyping of P. aeruginosa isolates. J.Cyst.Fibrosis 17, 729-735. doi: 10.1016/j.jcf.2018.03.004

Khosravi, A. D., Hoveizavi, H., Mohammadian, A., Farahani, A., and Jenabi, A. (2016). Genotyping of multidrug-resistant strains of Pseudomonas aeruginosa isolated from burn and wound infections by ERIC-PCR. Acta Cir.Bras.31, 206-211. doi: 10.1590/S0102-865020160030000009

Kocincova, D., Ostler, S. L., Anderson, E. M., and Lam, J. S. (2012). Rhamnosyltransferase genes migA and wapR are regulated in a differential manner to modulate the quantities of core oligosaccharide glycoforms produced by Pseudomonas aeruginosa. J.Bacteriol.194, 4295-4300. doi: 10.1128/ JB.05741-11

Kortright, K. E., Chan, B. K., Koff, J. L., and Turner, P. E. (2019). Phage therapy: a renewed approach to combat antibiotic-resistant bacteria. Cell Host Microbe 25, 219-232. doi: 10.1016/j.chom.2019.01.014

Lam, J. S., Taylor, V. L., Islam, S. T., Hao, Y., and Kocincova, D. (2011). Genetic and functional diversity of Pseudomonas aeruginosa lipopolysaccharide. Front.Microbiol.2:118. doi: 10.3389/fmicb.2011.00118

Le, S., He, X. S., Tan, Y. L., Huang, G. T., Zhang, L., Lux, R., et al. (2013). Mapping the tail fiber as the receptor binding protein responsible for differential host specificity of Pseudomonas aeruginosa bacteriophages PaP1 and JG004. PloS one 8:8. doi: 10.1371/journal.pone.0068562

Le, S., Yao, X., Lu, S., Tan, Y., Rao, X., and Li, M. (2014). Chromosomal DNA deletion confers phage resistance to Pseudomonas aeruginosa. Sci.Rep.4, 4738. doi: 10.1038/srep04738

Li, G., Shen, M., Yang, Y., Le, S., Li, M., and Wang, J. (2018). Adaptation of Pseudomonas aeruginosa to Phage PaP1 Predation via O-Antigen polymerase mutation. Front.Microbiol.9:1170. doi: 10.3389/fmicb.2018.01170

Lopez-Causape, C., Cabot, G., Del Barrio-Tofino, E., and Oliver, A. (2018). The versatile mutational resistome of Pseudomonas aeruginosa. Front.Microbiol.9:685. doi: 10.3389/fmicb.2018.00685

Lu, S. G., Le, S., Tan, Y. L., Zhu, J. M., Li, M., and Rao, X. C. (2013). Genomic and proteomic analyses of the terminally redundant genome of the Pseudomonas aeruginosa Phage PaP1: establishment of genus PaP1-Like Phages. PloS One 8:13. doi: 10.1371/journal.pone.0062933

Lu, S., Le, S., Li, G., Shen, M., Tan, Y., and Zhao, X. (2015). Complete genome sequence of Pseudomonas aeruginosa PA1, Isolated from a patient with a respiratory tract infection. Genome Announc.3:e01453-15.

Mantynen, S., Sundberg, L. R., and Poranen, M. M. (2018). Recognition of six additional cystoviruses: Pseudomonas virus phi6 is no longer the sole species of the family cystoviridae. Arch. Virol. 163, 1117-1124. doi: 10.1007/s00705-0173679-4
Paterson, S., Vogwill, T., Buckling, A., Benmayor, R., Spiers, A. J., and Thomson, N. R. (2010). Antagonistic coevolution accelerates molecular evolution. Nature 464, 275-278. doi: 10.1038/nature08798

Pires, D. P., Melo, L., Vilas, Boas D, Sillankorva, S., and Azeredo, J. (2017). Phage therapy as an alternative or complementary strategy to prevent and control biofilm-related infections. Curr. Opin. Microbiol. 39, 48-56. doi: 10.1016/j.mib. 2017.09.004

Roach, D. R., Leung, C. Y., Henry, M., Morello, E., Singh, D., and Di Santo, J. P. (2017). Synergy between the host immune system and bacteriophage is essential for successful phage therapy against an acute respiratory pathogen. Cell Host Microbe 22, 38.e-47.e.

Rohde, C., Resch, G., Pirnay, J. P., Blasdel, B. G., Debarbieux, L., and Gelman, D. (2018). Expert opinion on three phage therapy related topics: bacterial phage resistance, phage training and prophages in bacterial production strains. Viruses 10:E178.

Schwartz, D. A., and Lindell, D. (2017). Genetic hurdles limit the arms race between Prochlorococcus and the T7-like podoviruses infecting them. Isme J. 11, 1836-1851. doi: 10.1038/ismej.2017.47

Shen, M., Zhang, H., Shen, W., Zou, Z., Lu, S., and Li, G. (2018). Pseudomonas aeruginosa MutL promotes large chromosomal deletions through nonhomologous end joining to prevent bacteriophage predation. Nucleic Acids Res. 46, 4505-4514. doi: 10.1093/nar/gky160

Shen, M. Y., Le, S., Jin, X. L., Li, G., Tan, Y. L., and Li, M. (2016). Characterization and comparative genomic analyses of Pseudomonas aeruginosa Phage PaoP5: new members assigned to PAK_P1-like viruses. Sci.Rep.6, 6. doi: 10.1038/ srep34067

Smith, E. E., Buckley, D. G., Wu, Z. N., Saenphimmachak, C., Hoffman, L. R., and D'Argenio, D. A. (2006). Genetic adaptation by Pseudomonas aeruginosa to the airways of cystic fibrosis patients. Proc. Natl. Acad. Sci. U.S.A. 103, 8487-8492.

Sun, F. J., Shi, H. Q., Zhang, X. B., Fang, Y. D., Chen, Y. C., and Chen, J. H. (2013). Detection of carbapenemase-encoding genes among clinical Isolates of Pseudomonas aeruginosa in a chinese burn unit. J. Burn Care Res. 34, 453-458. doi: 10.1097/BCR.0b013e3182700afd

Waters, E. M., Neill, D. R., Kaman, B., Sahota, J. S., Clokie, M. R. J., Winstanley, C., et al. (2017). Phage therapy is highly effective against chronic lung infections with Pseudomonas aeruginosa. Thorax 72, 666-667. doi: 10.1136/thoraxjnl2016-209265

Waters, V., and Grimwood, K. (2018). Defining chronic Pseudomonas aeruginosa infection in cystic fibrosis. J.Cyst.Fibrosis 17, 292-293. doi: 10.1016/j.jcf.2018. 03.007

Yang, Y. H., Lu, S. G., Shen, W., Zhao, X., Shen, M. Y., and Tan, Y. L. (2016). Characterization of the first double-stranded RNA bacteriophage infecting Pseudomonas aeruginosa. Sci.Rep.6, 8.

Conflict of Interest: The authors declare that the research was conducted in the absence of any commercial or financial relationships that could be construed as a potential conflict of interest.

Copyright (c) 2020 Yang, Shen, Zhong, Chen, He, Baker, Xiong, Jin, Wang, Hu and Le. This is an open-access article distributed under the terms of the Creative Commons Attribution License (CC BY). The use, distribution or reproduction in other forums is permitted, provided the original author(s) and the copyright owner(s) are credited and that the original publication in this journal is cited, in accordance with accepted academic practice. No use, distribution or reproduction is permitted which does not comply with these terms. 